\title{
Influence of layer thickness on mechanical properties of multilayered NiFe samples
}

\section{processed by electrodeposition}

\author{
L. Kurmanaeva ${ }^{\mathrm{a}}$, J. McCrea ${ }^{\mathrm{b}}$, J. Jian ${ }^{\mathrm{c}}$, J. Fiebig ${ }^{\mathrm{a}}$, H. Wang ${ }^{\mathrm{c}}$, \\ A.K. Mukherjee ${ }^{\mathrm{a}}$, and E.J. Lavernia ${ }^{\mathrm{a}, \mathrm{d}}$ \\ a-Department of Chemical Engineering and Materials Science, University of California, Davis, CA 95817, USA \\ b- Integran Technologies Inc., Toronto, Canada \\ c- Department of Electrical and Computer Engineering, Texas A \& M University, College Station, TX 77843-3128, USA \\ d-Department of Chemical Engineering and Materials Science, University of California, Irvine, CA 92697
}

\begin{abstract}
s
Multilayered (ML) NiFe thick foils have been studied in order to provide in synthesizing structure-modulated sheets with superior mechanical properties. The specific electrodeposition parameters were selected to produce ML samples with modulated grain size distribution and layer thicknesses varied from $30 \mathrm{~nm}$ up to $5 \mu \mathrm{m}$. The influence of layer thickness on mechanical behavior was characterized by coupling nanoindentation tests and SEM/EDS study. Hardness results show that with decreasing layer thickness the strength of ML samples reaches a maximum value and remains relatively constant when layer thickness is less than $100 \mathrm{~nm}$. The measured properties are discussed in the context of layer configuration, overall dimensions, and change in phase and chemical composition.
\end{abstract}

Keywords: Ni-Fe, electrodeposition, multilayered structure, nanoindentation, EDS

\section{Introduction}

The ever increasing need to enhance the performance of devices and coatings has prompted research into strategies that can be implemented to decrease the dimensions of thin films and coatings without compromising their mechanical performance. The development of nanostructured metallic multilayers (NMM), for example, represents a successful strategy that has resulted in enhanced performance, such as high strength [1], improved tribological performance [2], giant magneto resistance [3], improved corrosion resistance [4,5], and enhanced thermal stability [6]. Survey of the published literature shows that in most NMM the layers contain different chemistries, with layer thickness values that are smaller than $50 \mathrm{~nm}$, as originally proposed in the pioneering work by Koehler [7]. In this study, Koehler argued that the materials for alternating layers should be selected to have nearly equal lattice parameter and 
thermal expansion values, but different elastic constants, so that strengthening can be ensured from a relative moduli mismatch.

Several methods have been employed to produce NMM, such as accumulative roll bonding [8], electrodeposition [3,9], magnetron sputtering [10], and vapor deposition [5]. Among these techniques, electrodeposition (ED) has emerged as an attractive approach, as a result of its versatility and low cost [11]. The first successful attempt to produce an electrodeposited composition-modulated material using two separated bath was reported by Brenner in 1939 [12]. However, such a method was cumbersome, because of the transfer operation required to be performed in a minimum time in order to prevent a surface passivation. In order to simplify the ED of NMM, in the 1980s, the usage of a single bath containing the salts of both components was proposed as a far more promising alternative [13]. The solution design of the single bath NMM should take into account several important principles: (1) two metals must be soluble and have solution compatibility at a preferred PH level, (2) two metals should have different reduction potentials [14]. In 1987 Yahalom and Zadok [13] reported the successful application of single bath ED to produce $\mathrm{Cu}-\mathrm{Ni} \mathrm{NMM}$, where modulation of layers was achieved down to 0.8 $\mathrm{nm}$ in each layer. Subsequent studies demonstrated that other metal pairs and metal alloys pairs could be deposited in a similar way from single baths, and Ross summarized an extended list of electrodeposited multilayer thin films in a review [15].

Recently a new type of electrodeposited NMM, in which alternative layers were synthesized to contain similar phase composition, was demonstrated. Such layer modulation was achieved by variation of grain size from $500 \mathrm{~nm}$ down to $16 \mathrm{~nm}$ in NiFe [9] and from $5 \mu \mathrm{m}$ down to $17 \mathrm{~nm}$ in $\mathrm{Ni}_{80} \mathrm{Co}$ [16]. The layers with nanocrystalline (NC) microstructures provided strength whereas coarser grained (CG) layers were introduced to increase the toughness and ductility of ML materials. The selection of a Ni-Fe alloy was motivated by several factors. First, Ni-Fe (Invar, Permalloy) deposits are widely used for decorative and protective coatings, or as undercoats in the steel making industry as well as for magnetic devices. Second, Fe-rich alloys provide benefits such as lower processing costs relative to those required in Ni rich plating, for example. Third, another important property of the Invar alloys with Fe content around 64 wt.\%. is a lower coefficient of thermal expansion (CTE), which makes this alloy an excellent choice for coating carbon fiber composites due to a low CTE mismatch with the substrate [17]. In this work, ML $\mathrm{Ni}-\mathrm{Fe}$ samples with various layer thicknesses were prepared and tested to explore challenges of 
ED processed modulated sheets and to study the influence of layer configuration and overall dimensions on mechanical behavior.

\section{Methods}

Multilayered (ML) and monolithic Ni-Fe samples were synthesized at Intergran Technology Inc. via a single bath electrodeposition technique from Integran Nanovar® $(\mathrm{Ni}-64 \% \mathrm{Fe})$ using a bath consisted of nickel sulfate, nickel chloride and ferrous sulfate at Integran Technologies Inc. (Toronto, Canada). The specific pulse plating parameters (change of electric waveform) were selected to produce monolithic or modulated microstructures (alternating NC and CG sub-layers) with a constant thickness ratio of 1:1, sub-layer thicknesses varied from $30 \mathrm{~nm}$ up to $5 \mu \mathrm{m}$ (ML $30 \mathrm{~nm}$, ML $50 \mathrm{~nm}$, ML $100 \mathrm{~nm}$, ML $300 \mathrm{~nm}$, ML $1 \mu \mathrm{m}$, ML $5 \mu \mathrm{m})$. The basic electrodeposition process to produce nanomaterials by ED has been previously described in patent [18]. Two different pulsed electrical waveforms were used in the process to produce the ML samples. The thickness of the individual layers were controlled by the deposition time at each condition. A programmable pulsed plating power supply was used to automatically switch between the two different pulsed waveforms at intervals that correlate to the deposition time required to achieve the desired layer thickness for each layer. Large sized sheets with a total thickness of about 500$600 \mu \mathrm{m}$ were produced by depositing onto a passivated Titanium or stainless steel cathodes from which they were subsequently mechanically stripped to yield free-standing samples.

The variations in hardness, elastic modulus and chemical composition were studied through

thickness, using rectangular shape specimens prepared using either Buhler Transoptic ${ }^{\mathrm{TM}}$ or ProbeMet ${ }^{\circledR}$ compound in a Buehler SimpliMet ${ }^{\circledR} 3000$ mounting equipment. The surfaces of studied samples were carefully polished using SiC sand papers, with further fine polishing using colloidal silica suspension Buehler MasterMet ${ }^{\circledR}$. Nanoindentation tests were performed using a Berkovich nanoindenter with a tip radius of $20 \mathrm{~nm}$ on the MTS nanoindenter XPS system. The maximum indentation depth was set up to be $2000 \mathrm{~nm}$ with a loading strain rate of $0.05 \mathrm{~s}^{-1}$, the maximum load held for $10 \mathrm{~s}$. Multiple indents were performed on every sample to evaluate variability. The error bars were estimated as standard deviation of all measured data for every sample. Information about hardness and values of the elastic modulus were measured during the unloading part of indentation. 
The as-deposited microstructure and chemical composition were studied using a FEI Scios DualBeam scanning electron microscope (SEM) with a voltage of 5-20 kV, equipped with an Oxford Nordlys detector. Energy dispersive spectroscopy (EDS) was used for an identification of chemical composition of sub-layers using line or point scanning. Since the content of minor impurities in ED NiFe sample was less than 0.15 wt.\% (including $0.026 \% \mathrm{C}$ and $0.018 \% \mathrm{~S}$ ) [9], during EDS analysis it was assumed sample contains Fe and Ni. In our study we present data on Fe content since Ni content can be calculated as (100 - xFe) wt.\%. Cross-sectional transmission electron microscopy (TEM) and scanning TEM (STEM) of samples with nano-sized layers (30$50 \mathrm{~nm}$ ) were performed using a FEI Tecnai G2 F20 analytical microscope operated under 200KV with a double-tilt holder and a point-to-point resolution of $0.24 \mathrm{~nm}$. An energy dispersive X-ray (EDX) spectroscopy of the samples was characterized by an EDX detector embedded in the microscope. TEM samples were prepared through a standard cross-section sample preparation process including sectioning, gluing, grinding, polishing, and final precision ion polishing.

\section{Results}

Figure 1 (a-b) presents results of nanoindentation tests of monolithic NC (filled squares) and CG sample (open circle), where the hardness and the elastic modulus were measured through cross section starting from the substrate side. In both samples, values for the measured hardness and elastic modulus were higher on the side close to substrate. However, in the monolithic NC sample the variation of hardness and elastic modulus through the thickness was less pronounced as compared to those obtained from the monolithic CG sample. We related the change in hardness and elastic modulus of the monolithic CG sample to the variation in Fe content through the thickness (Fig. 1c). For coarse grained material it was already reported by Li and Ebrahimi [19] that the change of Fe content from 40 to $60 \mathrm{wt} \% \%$ resulted in an increase of flow stress, which is in agreement with our results for CG NiFe. In the case of the monolithic NC material, the change of Fe content did not significantly change the hardness or elastic modulus. The average hardness and elastic modulus in the monolithic NC sample were 5.7 $\pm 0.1 \mathrm{GPa}$ and $130 \pm 7$ $\mathrm{GPa}$, respectively. The details of microstructure characterization of monolithic $\mathrm{NC}$ and $\mathrm{CG}$ samples can be found elsewhere [9]. From this work, the monolithic NC sample had a uniform microstructure with an average grain size of $16 \mathrm{~nm}$ and a mixture of fcc and bcc phases. Moreover, in the case of the monolithic CG sample the microstructure was noted to be elongated and oriented along ED direction with a $\langle 110\rangle$ texture. Therefore, it was not surprising that that 


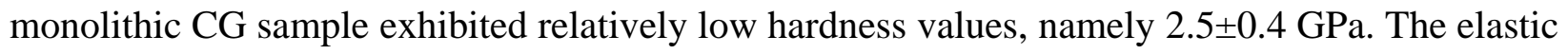
modulus of the monolithic CG sample was $162 \pm 15 \mathrm{GPa}$, which is higher than that corresponding to that of the monolithic NC sample. The average elastic modulus of Invar alloys (60-64 wt.\% $\mathrm{Fe}$ ) has been reported to be approximately $150 \mathrm{GPa}$ [20]; however, the elastic modulus is sensitive to changes in phase composition, chemical composition, texture, and etc.

Similar to characterization of the monolithic samples, the hardness and the elastic modulus were measured through thickness in every ML sample starting from the substrate side (Fig. 2 af), where the hardness data is presented as open square symbols (as left Y-axis) and the elastic modulus is presented by open triangle symbols (as right $\mathrm{Y}$-axis). The measured variations in nanoindentation values obtained for ML samples were similar to those obtained from the monolithic NC sample. With decreasing sub-layer thickness differences in hardness and elastic modulus from different sides of the samples decrease. Furthermore, a decrease of sub-layer thickness results in an increase in average hardness and a decrease of elastic modulus. Since the dimensions of the indents were around 7-10 $\mu \mathrm{m}$, in the multilayered sample with sub-layer thickness of $5 \mu \mathrm{m}(\mathrm{ML} 5 \mu \mathrm{m})$ indents covered only two layers with a variation of volume fraction of NC and CG microstructures, which could account for the increased standard deviation of measured data.

Figure 3a-c show representative SEM images obtained from the multilayered samples with layers thickness of $1 \mu \mathrm{m}$ (ML $1 \mu \mathrm{m}), 300 \mathrm{~nm}(\mathrm{ML} 300 \mathrm{~nm})$ and $100 \mathrm{~nm}$ (ML $100 \mathrm{~nm}$ ), respectively. The microstructure of ML $5 \mu \mathrm{m}$ sample was discussed in Ref [9], where we reported that there was a slight change in chemical composition between the sub-layers. In ML 1 $\mu \mathrm{m}$, ML $300 \mathrm{~nm}$ and ML $100 \mathrm{~nm}$ samples the sub-layers are distinct, as evident from the SEM images, due to differences in chemical composition (Fig 3d). The average sub-layer thicknesses in ML samples with layer thicknesses from $100 \mathrm{~nm}$ up to $5 \mu \mathrm{m}$ are close to the targeted thickness. With decreasing sub-layer thickness, the local thicknesses varied within $\pm 20 \%$ of the targeted values. The results of Fe composition of ML sample from EDS analysis are summarized in Table 1.

Figures 4 a-d show TEM and STEM micrographs of the cross section of multilayered samples with sub-layer thickness of $30 \mathrm{~nm}$ (ML $30 \mathrm{~nm}$ ) and $50 \mathrm{~nm}$ (ML $50 \mathrm{~nm}$ ), respectively. As evident from the dark field TEM images (Fig. 4a, 4c) both samples are characterized by uniform 
nanocrystalline microstructures with an average grain size of less than $30 \mathrm{~nm}$ in both sub-layers. Conventional TEM did not resolve differences between sub-layers, and there was no distinct sublayer with larger grain size. However, sub-layers still could be observed in STEM in high angle annular dark field (HAADF, also called Z-contrast) mode, where the image contrast is proportional roughly to $\mathrm{Z}^{2}$. In Fig. $4 \mathrm{~b}$ and $4 \mathrm{~d}$, it suggests that there are minor composition variations through the film sub-layers. Interestingly the ML $30 \mathrm{~nm}$ sample had more uniform sub-layer thickness in comparison with the $50 \mathrm{~nm}$ ML sample. The average thickness in ML $30 \mathrm{~nm}$ sample was approximately $40 \mathrm{~nm}$ (Fig. 4d), which is slightly larger than the targeted thickness. In ML $50 \mathrm{~nm}$ sample the thickness varied from $15 \mathrm{~nm}$ to $50 \mathrm{~nm}$ (Fig. 4b). Since layer thickness was so small, we were not able to characterize the chemical composition in sub-layers and therefore only the average overall Fe content in ML $30 \mathrm{~nm}$ and $50 \mathrm{~nm}$ samples is listed in Table 1. The ML $50 \mathrm{~nm}$ sample has the highest Fe content in compare to other samples.

\section{Discussion}

The basic mechanisms for the formation of the coherent polycrystalline deposit involves 3D crystalline growth or nucleation and coalescence growth with the following growth sequence: (1) formation of isolated nuclei and their 3D growth, (2) coalescence, (3) formation of linked network, (4) formation of continuous deposit [21]. Usually during ED a columnar microstructure is formed, composed of relatively fine grains near substrate and columnar coarse grains at greater distance away from the substrate. The monolithic CG sample presents a typical example of such a structure [9]. In order to obtain a nanocrystalline structure the nucleation rate of new grains should faster than the grain growth rate. Despite the fact that there are many variables in the ED process, there are only a few factors that may significantly influence the crystal structure and grain size of the deposits, such as a type of deposition (pulsing or direct current), applied negative overpotential, and an adion population at the electrode surface [22]. In order to produce a nanocrystalline structure a high negative overpotential, a high adions population, and low surface mobility of adions are required conditions for high nucleation rates, resulting in inhibition of grain growth. In our case, in order to form a layered structure the overpotential was varied through the use of pulsed current ED, which led to changes in the co-deposition rate. The family of Ni-Fe alloys belongs to a system that is well known for the occurrence of anomalous co-deposition [23], where the discharge rate of the more noble component (Ni) is inhibited, causing the appearance of the less noble component $(\mathrm{Fe})$ at a much higher ratio in the deposit 
than in the electrolyte, per se. Furthermore, the percentage of the less noble metal as a function of the applied potential goes through a maximum [23]. The last observation could explain our results since the elastic modulus and hardness values attained a maximum value on the substrate side (Fig. 1, Fig. 2). The increased deposition rate of Fe atoms led to changes in the Fe/Ni ratio in the bath and hence to depletion of Fe content in cross section of sample with further distance away from the substrate. Additionally we noticed that in some samples the indentations performed close to sample edges exhibited a significant drop in elastic modulus, which can be attributed to transient phenomena at the beginning and end of the deposition process [24]. The iron content is high on the substrate side and decreases during the deposition process due to edge effects, when dendrites growing at the edges of the samples lower the overall current density, which has an effect on composition.

As previously mentioned, the elastic modulus is sensitive to chemical composition variations; however, it is not usually dependent on structural parameters. However, it was shown in several $\mathrm{Ni}$ and Ni-Fe sample produced by ED that at grain size below $20 \mathrm{~nm}$ the texture and grain size influence the elastic modulus [25]. In present work, we noticed the variation in elastic modulus results in ML samples (Fig. 5a) due to changes in chemical and phase composition, which are partially caused by the decrease of sub-layer thickness. The elastic moduli of ML samples and monolithic NC sample were in the range of 125-140 GPa, which is lower than the elastic modulus of typical Invar alloys containing 64 wt.\% Fe, 141-178 GPa [26]. In our previous work [9] it was revealed by that the structure of monolithic NC and ML $5 \mu \mathrm{m}$ consists of the mixture of fcc and bcc phases. Based on our previous experiments [9] and recent TEM studies of monolithic NC and ML samples we were unable to distinguish the bcc phase on bright-field or dark-filed TEM images. As evident from Fig. 4 the contrast of nanocrystalline grains is serrated, which suggests that the fcc to bcc phase transformation could be stress driven, and similar to martensitic transformation. High internal stresses are known to be common during ED of NC systems and can sometimes lead to the formation of cracks in as-deposited materials [22]. It is possible that the cycling of process parameters, that are required to generate a layered structure in the present experiments, may have contributed to the development of high internal residual stresses. For instance, XRD analysis of $\mathrm{Fe} / \mathrm{Cu} \mathrm{ML}$ material confirmed that layer interface were highly strained, which led to in-plane magnetic anisotropy of Fe layer [27]. In NiFe ML sample there is one more possible source of the increased internal stresses along interfaces, which is the 
decreased thermal expansion coefficient of the NC sub-layers. To that effect, it was reported by Grimmet et al. [28] that the presence of bcc phase significantly changed the CTE of NiFe alloys with 50-60 wt.\% Fe due to increased presence of the bcc phase.

Control of epitaxial growth plays an important role in the formation and uniformity of the multilayered structures. When switching ED regime from the CG to NC layers, the new layer prefers to continue growing within the existing structure. In recent work different pulse conditions were used in order to increase the nucleation rate and change in grain size. There is a transition period between the layers to be able to "seed" the new microstructure. For example with the ML NiCo [16], it was unable to produce ML structures with layers smaller than $500 \mathrm{~nm}$ layer thickness, due to large transition period. In case of ML NiFe the presence of bcc phase could be one of the key factors that result in forming ML structure at the fine scale down to 30 $\mathrm{nm}$. Since the CG layer is primarily fcc, we suppose that the presence of the BCC phase in transition layer promotes nucleation of distinct NC layer with a much smaller transition period as compared with NiCo system.

Nanoindentation tests of ML samples confirmed that the decrease of sub-layer thickness hardens ML materials (Fig. 2). Similar to bulk materials, the strengthening of NMM with layer thickness in sub-micron range follows a Hall-Petch type relationship: $\sigma \sim \sqrt{ } \mathrm{h}$, where stress $(\sigma)$ is inversely proportional to the root square of layer thickness (h) [29,30]. It is assumed that in this range $\mathrm{h}>100 \mathrm{~nm}$ the deformation mechanism of ML samples is controlled by the glide of single dislocations confined within individual layers. Figure 5b summarizes results from figure 2 in a plot "hardness vs. inverse square root of sub-layer thickness", where the range of sub-layer thickness above $100 \mathrm{~nm}$ are fitted with linear function, meaning that it follows afore mentioned Hall-Petch type relationship. Within the thickness range from $100 \mathrm{~nm}$ up to $5 \mu \mathrm{m}$ the ML sample are strengthened due to (1) decrease of the average grain size, (2) increase of the volume fraction of layer interfaces, (3) the change in chemical composition (higher volume fraction of bcc phase results in increase Peierls-Nabarro forces). The maximum hardness was achieved in ML $100 \mathrm{~nm}$ sample, which is slightly higher than hardness of monolithic NC sample, 5.9 $\pm 0.2 \mathrm{GPa}$ and $5.7 \pm$ $0.1 \mathrm{GPa}$, respectively. The average hardness of ML sample with finer layer thickness $(30 \mathrm{~nm}$ and $50 \mathrm{~nm}$ ) did not increase further following the Hall-Petch type relationship (Hall-Petch "break down”) and stayed within an error bar in comparison to ML $100 \mathrm{~nm}$ and monolithic NC samples. In a monocrystalline ML materials, when single layer presents almost a perfect single crystal, the 
"break down" of Hall-Petch type relationship was usually observed when layer thickness was below $50 \mathrm{~nm}[1,31,32]$. For example, in the study of mechanical properties of $\mathrm{ML} \mathrm{Cu}-\mathrm{X}(\mathrm{X}=\mathrm{Ni}$, $\mathrm{Nb}, \mathrm{Cr}$ ) the "break down" was indicated when layer thicknesses were as small as $10 \mathrm{~nm}$ [32]. It should be noted here, that in monocrystalline ML materials with layer thickness as small as few $\mathrm{nm}$ the deformation is still controlled by dislocation movement: a dislocations glide within layers or across layer interphases [1,33]. In the present work, the "break down" of Hall-Petch type relationship observed when layer thickness was around $100 \mathrm{~nm}$ due to the polycrystalline structure of layers. In case of polycrystalline ML sample, the deformation is controlled by NC sub-layers, where the average grain size was approximately $16 \mathrm{~nm}$ [9]. Due to the activation of grain boundary mediated deformation mechanisms in the nanocrystalline materials with average grain size below $30 \mathrm{~nm}$ [34], no strengthening was observed in ML sample with fine layers, and the average hardness of ML $30 \mathrm{~nm}$ and ML $50 \mathrm{~nm}$ samples was close to that of the monolithic NC sample.

\section{Summary}

Nanostructured multilayered $\mathrm{NiFe}$ samples with various layer thicknesses starting from $5 \mu \mathrm{m}$ down to $30 \mathrm{~nm}$ were successfully prepared by pulsed electrodeposition (ED) from single bath. A layered structure was maintained even in layers with a minimum thickness as fine as $30 \mathrm{~nm}$ due to a modulation of grain size and chemical composition. Nanoindentation tests along the cross section of ED sheets revealed an inhomogeneity of properties through thickness, which became less pronounced in samples with finer sub-layers. With decreasing of sub-layer thickness the hardness of multilayered samples reached the maximum of $5.9 \mathrm{GPa}$ in the sample with 100nm layer thickness and further reached a plateau when layer thickness was less than $100 \mathrm{~nm}$. The modification of processing parameters in order to prepare fine layers resulted in a variation of the chemical composition, which led to the change in elastic modulus. Elastic moduli of multilayered samples were lower than that of typical Invar alloys with 64 wt. \% Fe (141-148 $\mathrm{GPa}$ ) because of the presence of bcc phase, formed by stress induced phase transformation. 


\section{Acknowledgments}

Financial support from Office of Naval Research under grants N00014-13-1-0557 (Research at UCD), N00014-13-1-0555 (TEM work at Texas A\&M University), and N00014-10-C-0371

(Sample preparation at Integran Technologies Inc) is gratefully acknowledged. The authors are grateful to Mr. Hanry Yang (UC Davis) for the help in EDM machining of samples.

\section{References}

[1] J. Wang, A. Misra, Curr. Opin. Solid State Mater. Sci. 15 (2011) 20-28.

[2] S. Izadi, H. Mraied, W. Cai, Surf. Coat. Technol. 275 (2015) 374-383.

[3] I. Bakonyi, L. Péter, Prog. Mater. Sci. 55 (2010) 107-245.

[4] L.A.S. Ries, D.S. Azambuja, I.J.R. Baumvol, Surf. Coat. Technol. 89 (1997) 114-120.

[5] D. Zhou, H. Peng, L. Zhu, H. Guo, S. Gong, Surf. Coat. Technol. 258 (2014) 102-107.

[6] I.J. Beyerlein, J.R. Mayeur, R.J. McCabe, S.J. Zheng, J.S. Carpenter, N.A. Mara, Acta Mater. 72 (2014) 137-147.

[7] J.S. Koehler, Phys. Rev. B 2 (1970) 547-551.

[8] D. Bhattacharyya, N.A. Mara, P. Dickerson, R.G. Hoagland, A. Misra, J. Mater. Res. 24 (2009) 1291-1302.

[9] L. Kurmanaeva, H. Bahmanpour, T. Holland, J. McCrea, J.H. Lee, J. Jian, H. Wang, E.J. Lavernia, A.K. Mukherjee, Philos. Mag. 94 (2014) 3549-3559.

[10] R. Banerjee, S.A. Dregia, H.L. Fraser, Acta Mater. 47 (1999) 4225-4231.

[11] A. Ollagnier, P. Benaben, Met. Finish. 103 (2005) 56-63.

[12] A. Brenner, The Electrodeposition of Copper-Bismuth Alloys from a Perchlorate Bath, $\mathrm{PhD}$ thesis, University of Maryland, 1939.

[13] J. Yahalom, O. Zadok, J. Mater. Sci. 22 (1987) 499-503.

[14] D.R. Gabe, G.D. Wilcox, Met. Finish. 100 (2002) 18-27.

[15] C.A. Ross, Annu. Rev. Mater. Sci. 24 (1994) 159-188.

[16] M. Daly, J.L. McCrea, B.A. Bouwhuis, C.V. Singh, G.D. Hibbard, Mater. Sci. Eng. A 641 (2015) 305-314.

[17] J.L. McCrea, G. Palumbo, G.D. Hibbard, U. Erb, Rev. Adv. Mater. Sci. 5 (2013) 252-258.

[18] U. Erb, A.M. El-Sherik, Nanocrystalline Metals and Process of Producing the Same, US Pat. 5, 352, 266, 1994.

[19] H. Li, F. Ebrahimi, Mater. Sci. Eng. A 347 (2003) 93-101.

[20] W. Martienssen, H. Warlimont, Springer Handbook of Condensed Matter and Materials Data, Springer Science \& Business Media, 2006.

[21] M. Schlesinger, M. Paunovic, Modern Electroplating, John Wiley \& Sons, 2011.

[22] C.C. Koch, J. Phys. Conf. Ser. 144 (2009) 012081.

[23] A. Brenner, Electrodeposition of Alloys: Principles and Practice, Elsevier, 2013.

[24] S. Djokic, Electrodeposition and Surface Finishing: Fundamentals and Applications, Springer Science \& Business Media, 2014.

[25] J.D. Giallonardo, U. Erb, K.T. Aust, G. Palumbo, Philos. Mag. 91 (2011) 4594-4605.

[23] Material Property Data: http://www.matweb.com/

[27] E. Jartych, D. Chocyk, M. Budzyński, M. Jałochowski, Appl. Surf. Sci. 180 (2001) 246254. 
[28] D.L. Grimmett, M. Schwartz, K. Nobe, J. Electrochem. Soc. 140 (1993) 973-978.

[29] G.S. Was, T. Foecke, Thin Solid Films 286 (1996) 1-31.

[30] P.M. Anderson, C. Li, Nanostructured Mater. 5 (1995) 349-362.

[31] B.M. Clemens, H. Kung, S.A. Barnett, MRS Bull. 24 (1999) 20-26.

[32] A. Misra, M. Verdier, Y.C. Lu, H. Kung, T.E. Mitchell, M. Nastasi, J.D. Embury, Scr. Mater. 39 (1998) 555-560.

[33] P.M. Anderson, T. Foecke, P.M. Hazzledine, MRS Bull. 24 (1999) 27-33.

[34] Y. Ivanisenko, L. Kurmanaeva, J. Weissmueller, K. Yang, J. Markmann, H. Rösner, T. Scherer, H.-J. Fecht, Acta Mater. 57 (2009) 3391-3401. 
Table 1 Variation of Fe content in monolithic coarse-grained (CG), monolithic nanocrystalline (NC) and multilayered samples

\begin{tabular}{|c|c|c|c|c|}
\hline \multirow{2}{*}{\multicolumn{2}{|c|}{ Sub-layer thickness $(\mathrm{nm})$}} & \multirow{3}{*}{$\begin{array}{c}\text { Fe content (wt.\%) } \\
63 \pm 1.5\end{array}$} & \multicolumn{2}{|c|}{ Fe content in sub-layers (wt.\%) } \\
\hline & & & CG & $\mathrm{NC}$ \\
\hline \multirow{2}{*}{ 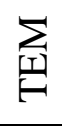 } & 30 & & - & - \\
\hline & 50 & $67 \pm 2$ & - & - \\
\hline \multirow{6}{*}{$\sum_{\mathbb{U}}$} & 100 & $63 \pm 1.5$ & $61.5 \pm 0.5$ & $63.1 \pm 0.5$ \\
\hline & 300 & $61 \pm 1.5$ & $60.6 \pm 0.5$ & $63.3 \pm 0.5$ \\
\hline & 1000 & $60 \pm 1.5$ & $57.5 \pm 0.5$ & $60.9 \pm 0.5$ \\
\hline & $5000 *$ & $61 \pm 1.5$ & $59.7 \pm 0.5$ & $62.8 \pm 0.5$ \\
\hline & mono CG & $53 \pm 10$ & \multicolumn{2}{|c|}{ no layers } \\
\hline & mono NC & $57 \pm 5$ & \multicolumn{2}{|c|}{ no layers } \\
\hline
\end{tabular}

*from ref. [9]

\section{Figure caption}

Figure 1 Variation of hardness (a), elastic modulus, (b) and Fe composition (c) in monolithic coarse grained and nanocrystalline samples across the thickness starting from the substrate side.

Figure 2 Variation of hardness and elastic modulus for multilayered samples with different sublayer thickness: (a) $5 \mu \mathrm{m}$, (b) $1 \mu \mathrm{m}$, (c) $300 \mathrm{~nm}$, (d) $100 \mathrm{~nm}$, (e) $50 \mathrm{~nm}$, (f) $30 \mathrm{~nm}$.

Figure 3 SEM micrographs of the cross section of multilayered (ML) sample with sub-layer thickness (a) $1 \mu \mathrm{m}$, (b) $300 \mathrm{~nm}$, and (c) $100 \mathrm{~nm}$. (d) EDS-SEM line scan across several layers in ML $1 \mu \mathrm{m}, 300 \mathrm{~nm}$ and $100 \mathrm{~nm}$ samples.

Figure 4 Dark field and scanning TEM micrographs of multilayered sample with sub-layer thickness $50 \mathrm{~nm}(\mathrm{a}, \mathrm{c})$ and $30 \mathrm{~nm}(\mathrm{~b}, \mathrm{~d})$

Figure 5 (a) Average elastic modulus vs. sub-layer thickness for sample with layer thickness from $30 \mathrm{~nm}$ up to $5 \mu \mathrm{m}$ samples. (b) Average hardness vs inverse square root of sub-layer thickness, h. Solid line represents the hardness level of monolithic nanocrystalline samples. Dotted line presents approximate function "Hardness $\sim 1 / \sqrt{ } \mathrm{h}$ " 

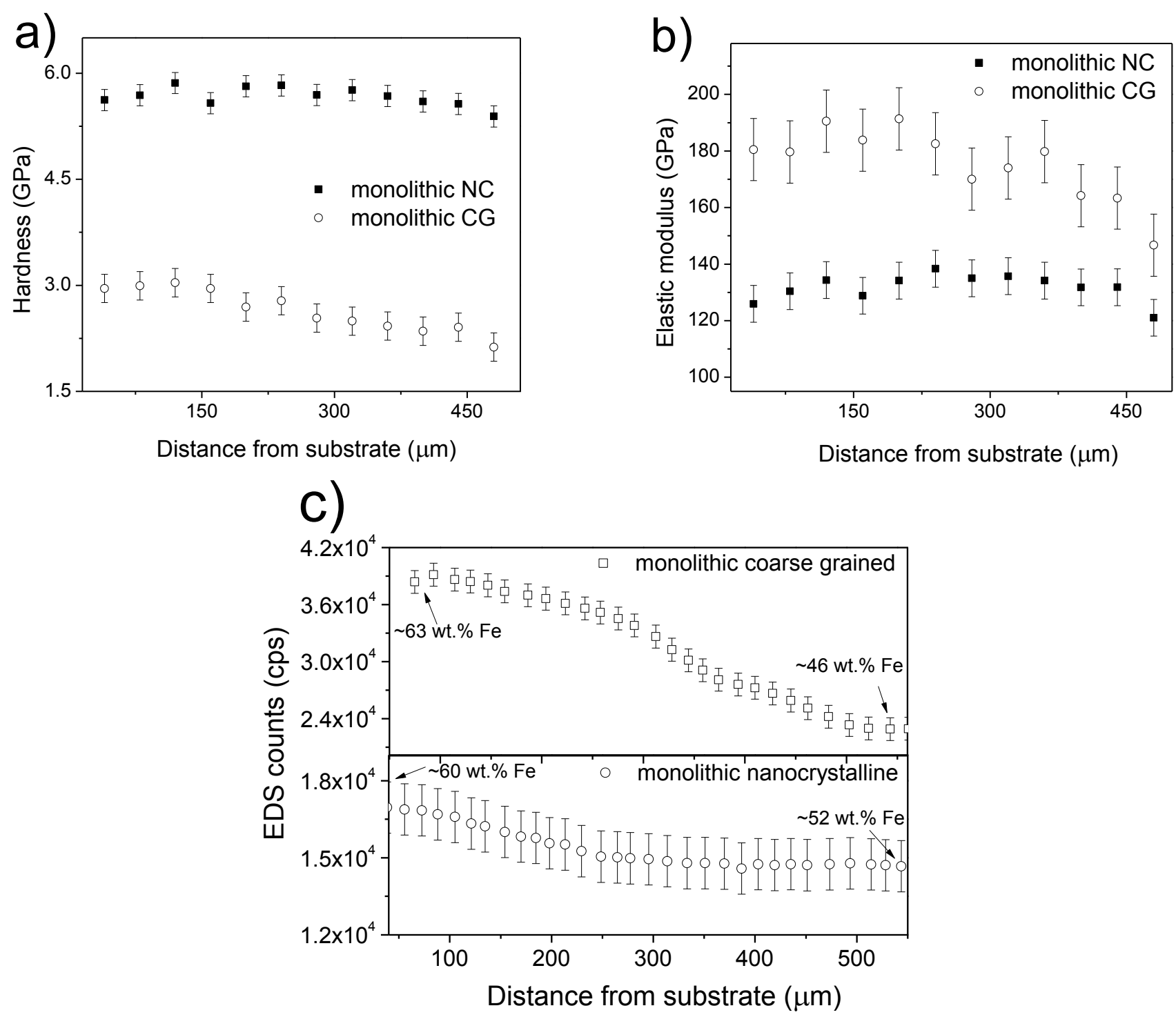

Figure 1 Variation of hardness (a), elastic modulus(b) and Fe composition (c) in monolithic coarse grained and nanocrystalline samples across the thickness starting from the substrate side 

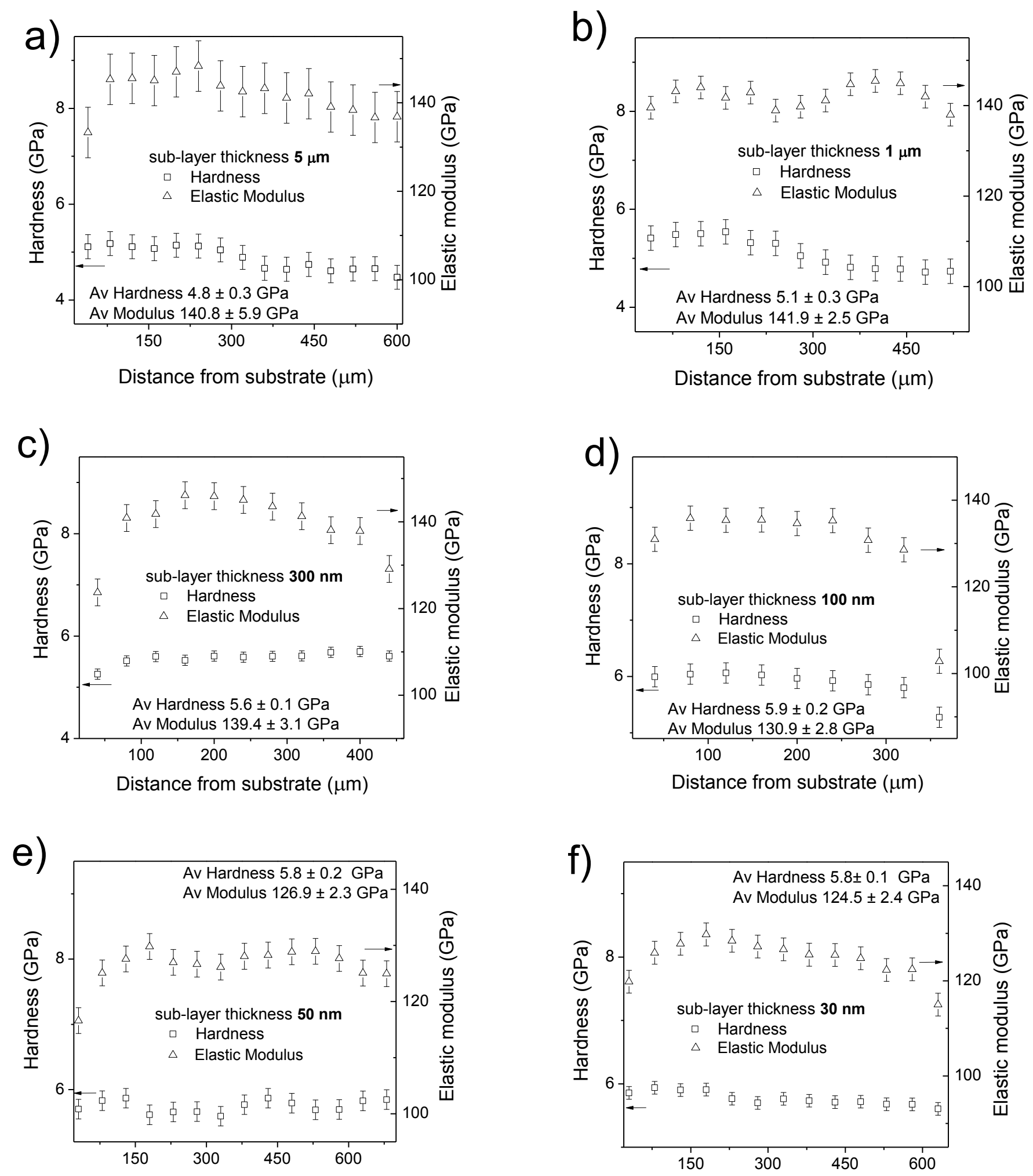

Distance from substrate $(\mu \mathrm{m})$

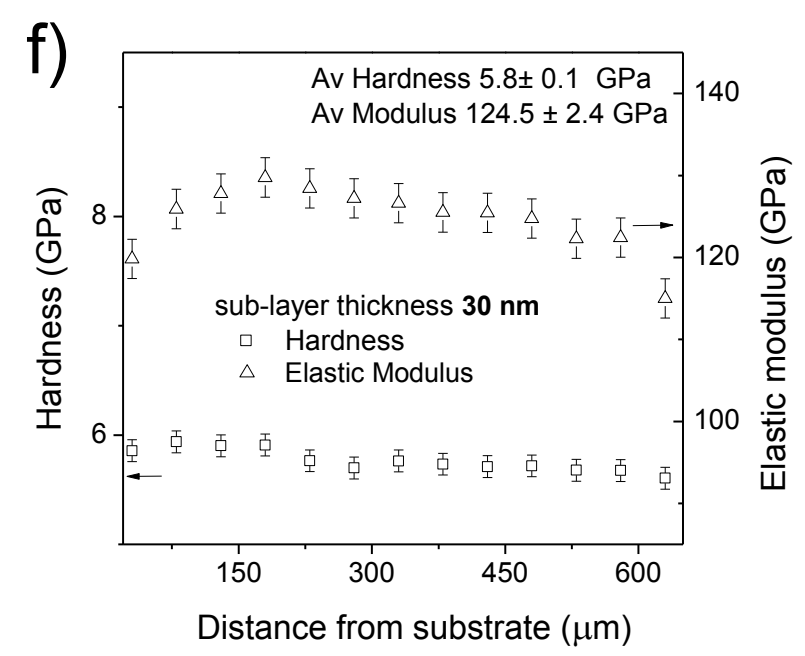

Figure 2 Variation of hardness and elastic modulus for multilayered samples with different sub-layer thickness: (a) $5 \mu \mathrm{m}$, (b) $1 \mu \mathrm{m}$, (c) $300 \mathrm{~nm}$, (d) $100 \mathrm{~nm}$, (e) $50 \mathrm{~nm}$, (f) $30 \mathrm{~nm}$ 


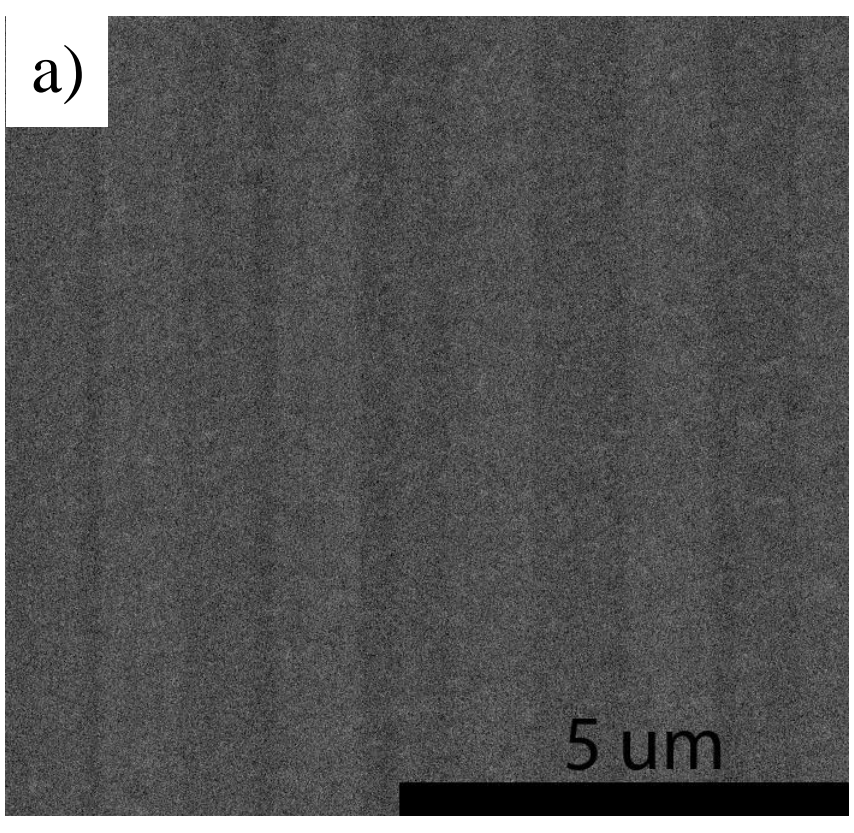

\section{b)}
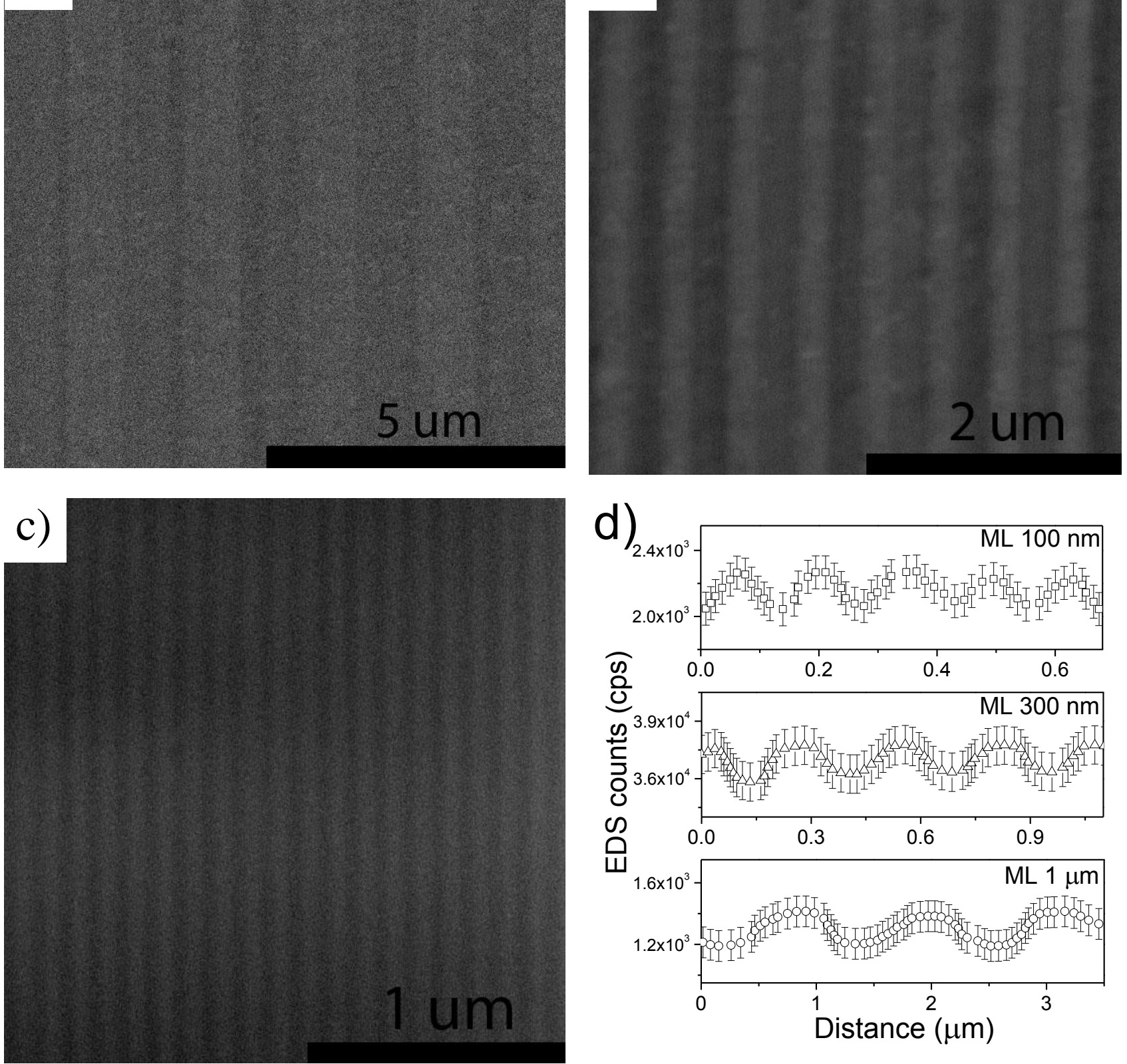

Figure 3 SEM micrographs of the cross section of multilayered (ML) sample with sub-layer thickness (a) $1 \mu \mathrm{m}$, (b) $300 \mathrm{~nm}$, and (c) $100 \mathrm{~nm}$. (d) EDS-SEM line scan across several layers in ML $1 \mu \mathrm{m}, 300$ $\mathrm{nm}$ and $100 \mathrm{~nm}$ samples. 

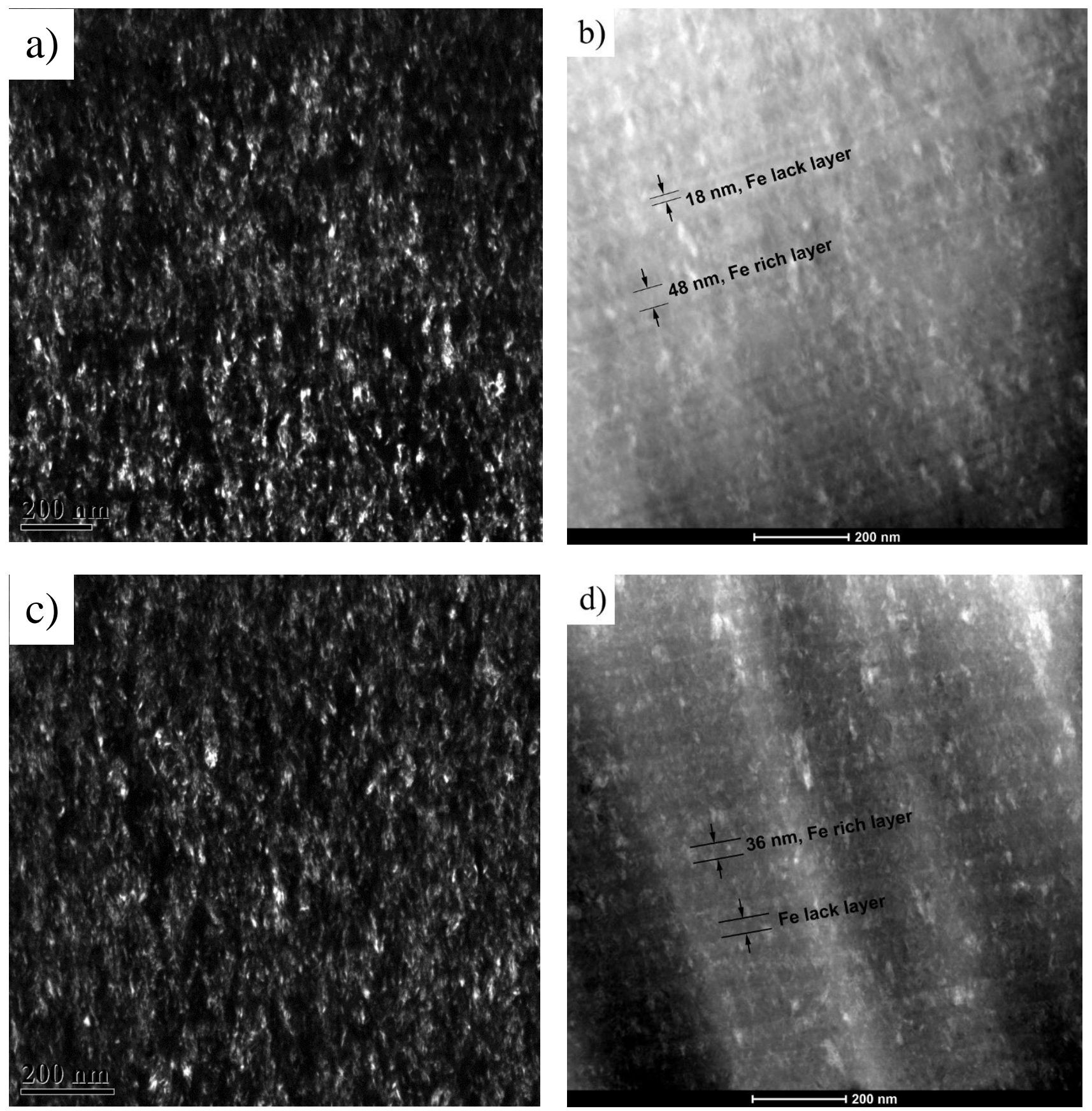

Figure 4 Dark field and scanningTEM micrographs of multilayered sample with sub-layer thickness $50 \mathrm{~nm}(\mathrm{a}, \mathrm{c})$ and $30 \mathrm{~nm}(\mathrm{~b}, \mathrm{~d})$ 

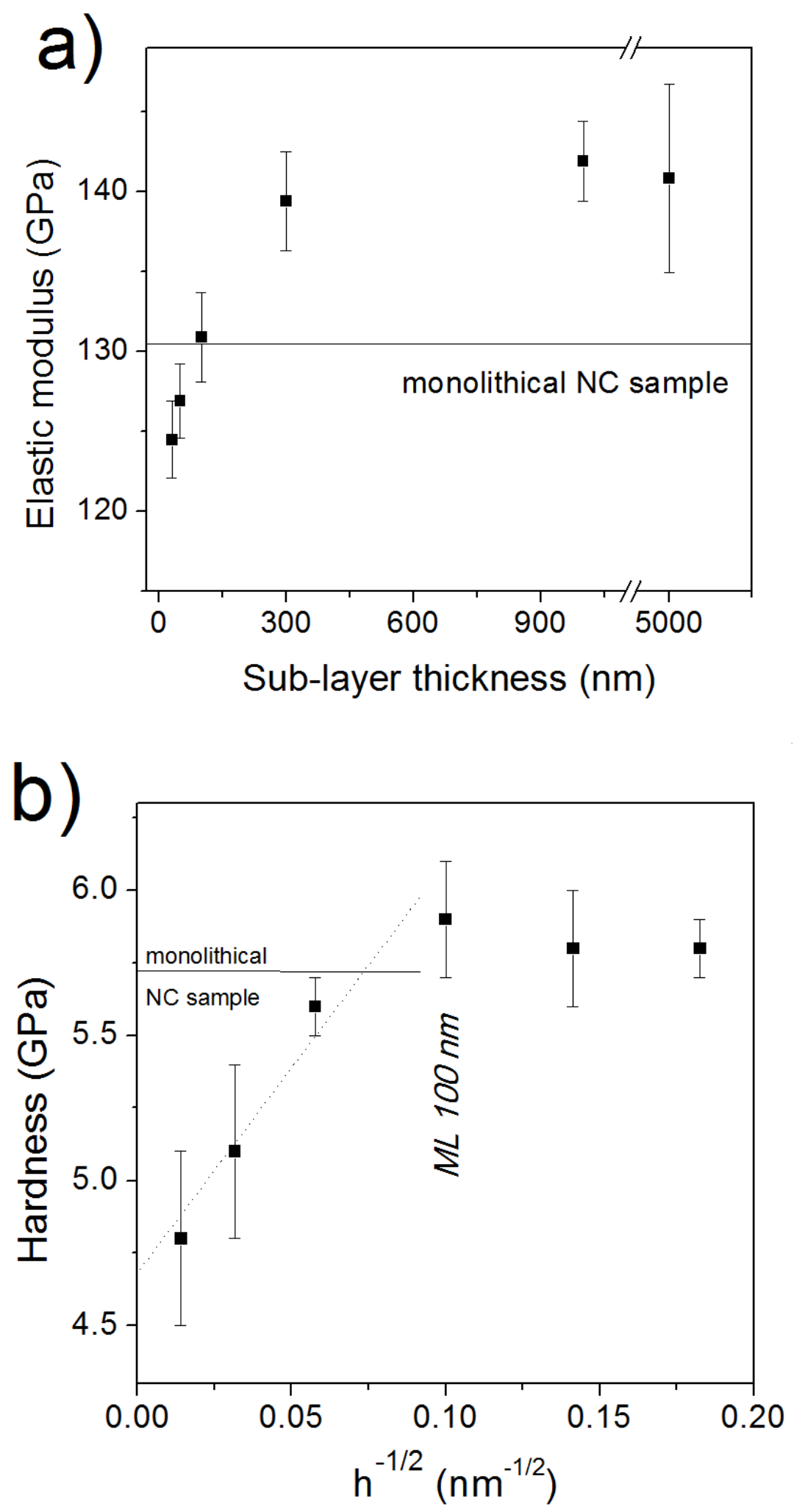

Figure 5 (a) Average elastic modulus vs. sub-layer thickness for sample with layer thickness from $30 \mathrm{~nm}$ up to $5 \mu \mathrm{m}$ samples. (b) Average hardness vs inverse square root of sub-layer thickness, h. Solid line represents the hardness level of monolithic nanocrystalline samples. Dotted line presents approximate function "Hardness 1/ $\sqrt{ } \mathrm{h} "$ 
